\title{
A Demonstration of Dynamic Drawing Guidance via Electromagnetic Haptic Feedback
}

\author{
Thomas Langerak \\ Department of Computer \\ Science \\ ETH Zürich \\ Zürich, Switzerland \\ thomas.langerak@inf.ethz.ch
}

\author{
Juan Zarate \\ Department of Computer \\ Science \\ ETH Zürich \\ Zürich, Switzerland \\ juan.zarate@inf.ethz.ch
}

\author{
Velko Vechev \\ Department of Computer \\ Science \\ ETH Zürich \\ Zürich, Switzerland \\ velko.vechev@inf.ethz.ch
}

\author{
Daniele Panozzo \\ Courant Institue of \\ Mathematical Sciences \\ New York University \\ New York,USA \\ panozzo@nyu.edu
}

\author{
Otmar Hilliges \\ Department of Computer \\ Science \\ ETH Zürich \\ Zürich, Switzerland \\ otmar.hilliges@ethz.ch
}

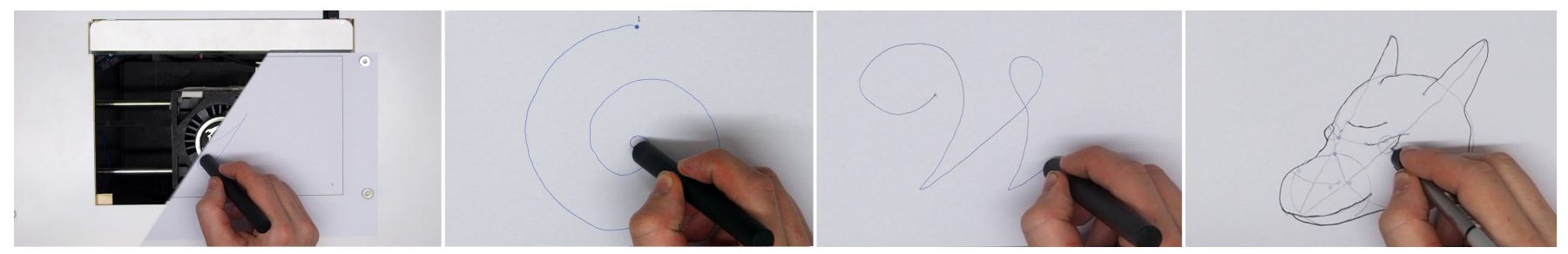

Figure 1: Left-to-right: an electromagnet moving on a bi-axial linear stage underneath a high-speed pressure sensitive tablet delivers dynamically adjustable haptic feedback to a minimally instrumented pen. This allows for in-situ haptic feedback aiding users in drawing primitive shapes, or in writing characters, and gives guidance in more complex sketching tasks. We experimentally show that our approach increases accuracy and may help in skill acquisition.

\begin{abstract}
We demonstrate a system to deliver dynamic guidance in drawing, sketching and handwriting tasks via an electromagnet moving underneath a high refresh rate pressure sensitive tablet presented in [3]. The system allows the user to move the pen at their own pace and style and does not take away control. Using a closed-loop time-free approach allows for error-correcting behavior. The user will experience to be smoothly and natural pulled back to the desired trajectory rather than pushing or pulling the pen to a continuously advancing setpoint. The optimization of the setpoint with regard to the user is unique in our approach.
\end{abstract}

Permission to make digital or hard copies of part or all of this work for personal or classroom use is granted without fee provided that copies are not made or distributed for profit or commercial advantage and that copies bear this notice and the full citation on the first page. Copyrights for third-party components of this work must be honored. For all other uses, contact the owner/author(s).

UIST' 19 Adjunct, October 20-23, 2019, New Orleans, LA, USA.

Copyright is held by the author/owner(s).

ACM ISBN 978-1-4503-6817-9/19/10.

http://dx.doi.org/10.1145/3332167.3356889

\section{Author Keywords}

hardware; haptics; magnetism; stylus-based interaction

\section{INTRODUCTION}

Sketches and handwritten text have been a primary form of communication for centuries. Given their importance for society at large and the arts and design in particular, it is not surprising that a large number of digital pen interfaces exist that aim to combine the expressiveness and flexibility of pens and pencils with the advantages of digital representations via standalone tablets (e.g., Apple iPad, Microsoft Surface) or specialized digitizers (e.g., Wacom). Several sketch [7, 5, 4, 6] and digital ink $[1,9]$ beautification approaches exist. However, these typically improve results a-posteriori and hence do not provide real-time feedback. Comparatively little attention has been devoted to improving sketching in-situ.

We demonstrate a haptic feedback system (Fig. 2) to provide variable strength guidance onto the tip of a minimally instrumented ballpoint pen. The feedback is delivered via an electromagnet moving on a bi-axial linear stage below a touch 


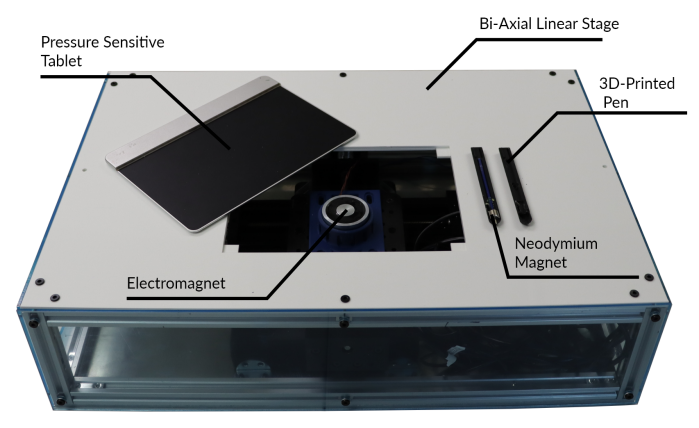

Figure 2: Hardware overview.

and pressure sensitive digital tablet, governed by a closed-loop optimal control algorithm. Our approach allows users to perceive different levels of feedback while drawing on the tablet. Importantly, the variable strength of force feedback is crucial in allowing user autonomy, while maintaining the ability to provide guidance.

Previous approaches to pen-based haptic feedback rely on permanent Neodymium magnets (e.g., [8]). However, due to the steep increase in magnetic force as the pen approaches the magnet, this fully controls the pen, removing agency of the user. In contrast we propose a novel optimization scheme, inspired by model predictive contour control (MPCC) [2], to position and regulate an electromagnet such that it provides dynamically adjustable in-plane magnetic forces to the pen tip. Using this optimization scheme allows for a large degree of user autonomy, which is in sharp contrast with DePENd [8] (in which the system does not react to user input). This autonomy enables, for instance, creative freedom (Figure 3). More technical details on the implementation of the control method can be found in [3].

\section{SYSTEM}

MagPen (Figure 2) is a compact, non-invasive tabletop haptic feedback system in which users draw on normal paper with a minimally modified ballpoint pen. The strokes are recorded by a Sensel Morph (https://sensel.com/) pressure sensitive touch pad. We chose the Sensel board for it's high spatial resolution $(6502 \mathrm{DPI})$, high speed $(500 \mathrm{~Hz})$ and low latency ( $2 \mathrm{~ms})$. Since the board is pressure sensitive, the sketching surface does not interfere with the input recognition. Users draw with a 3D printed ballpoint pen with a permanent ring magnet mounted in the shaft.

To deliver haptic feedback to the pen, we move a programmatically controlled electromagnet (Intertec ITS-MS-5030$12 \mathrm{VDC}$, diameter $=5 \mathrm{~cm}$, height $=3 \mathrm{~cm}, 12 \mathrm{~V} 11 \mathrm{~W}$ ) on a biaxial linear stage directly underneath the input sensor. Our implementation consists of a the bi-axial system and hightourque stepper motors.

\section{APPLICATIONS}

\section{Calligraphy:}

Figure 1 illustrate writing of flourished characters, with only minimal visual guidance (single starting point).

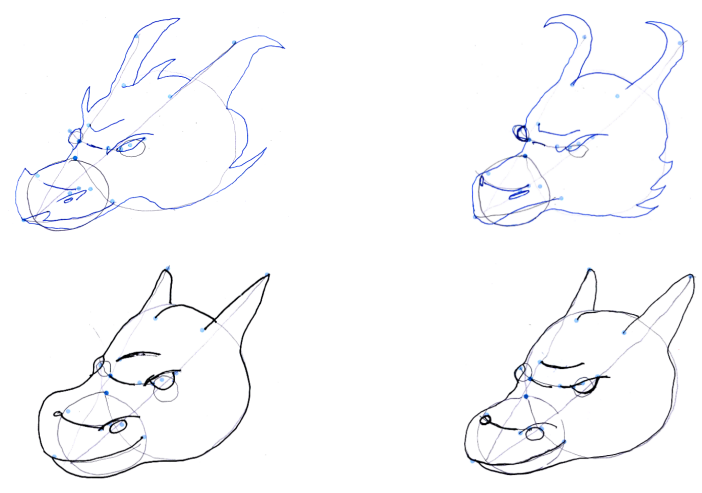

Figure 3: Different variants of the same dragon, drawn with identical system settings. Each pair of drawings used with different tools.

\section{Drawing teaching aid:}

Connect-the-dots exercises are often used to teach children motor skills as well as stroke ordering. Our original paper [3] shows results from a similar exercise performed with our system.

\section{Outlining \& inking:}

Figure 3 illustrates the effect of two core capabilities of the proposed approach. Here we first outline the proportions of the dragon head and then use different pens to ink-in the details. Note that the system provides haptic guidance but allows the user to draw the shape in different styles and with varying highfrequency detail, while maintaining similarity to the reference shape.

\section{DEMONSTRATION}

In our demonstration visitors will be able to draw a predetermined shape. During the drawing they will be encouraged to make mistakes on purpose to fully explore the hardware capabilities. To understand the system only takes 30 seconds, a simple drawing can take up to 3 minutes. Visitors can take the drawings home as souvenir. During the demo they will notice how to feedback increases with the error they are making. Next to this they will notice the smooth correction.

\section{ACKNOWLEDGEMENTS}

This work was supported in part by a grants from the Hasler Foundation (Switzerland), ERC (OPTINT StG-2016-717054) and NSF (CAREER 1652515 and OAC 1835712).

\section{REFERENCES}

[1] Emre Aksan, Fabrizio Pece, and Otmar Hilliges. 2018. DeepWriting: Making Digital Ink Editable via Deep Generative Modeling. In SIGCHI Conference on Human Factors in Computing Systems (CHI'18). ACM, New York, NY, USA.

[2] Denise Lam, Chris Manzie, and Malcolm Good. 2010. Model predictive contouring control. In Decision and Control (CDC), 2010 49th IEEE Conference on. IEEE, 6137-6142. 
[3] Thomas Langerak, Juan Jose Zarate, Velko Vechev, Daniele Panozzo, and Otmar Hilliges. 2019. Dynamic Drawing Guidance via Electromagnetic Haptic Feedback. CoRR abs/1906.11753 (2019). http://arxiv.org/abs/1906.11753

[4] Alex Limpaecher, Nicolas Feltman, Adrien Treuille, and Michael Cohen. 2013. Real-time drawing assistance through crowdsourcing. ACM Transactions on Graphics (TOG) 32, 4 (2013), 54.

[5] Edgar Simo-Serra, Satoshi Iizuka, Kazuma Sasaki, and Hiroshi Ishikawa. 2016. Learning to simplify: fully convolutional networks for rough sketch cleanup. ACM Transactions on Graphics (TOG) 35, 4 (2016), 121.

[6] Qingkun Su, Wing Ho Andy Li, Jue Wang, and Hongbo Fu. 2014. EZ-sketching: three-level optimization for error-tolerant image tracing. ACM Transactions on Graphics 33, 4 (2014).

[7] Jun Xing, Li-Yi Wei, Takaaki Shiratori, and Koji Yatani. 2015. Autocomplete hand-drawn animations. ACM Transactions on Graphics (TOG) 34, 6 (2015), 169.

[8] Junichi Yamaoka and Yasuaki Kakehi. 2013. dePENd: augmented handwriting system using ferromagnetism of a ballpoint pen. In Proceedings of the 26th annual ACM symposium on User interface software and technology. ACM, 203-210.

[9] C Lawrence Zitnick. 2013. Handwriting beautification using token means. ACM Transactions on Graphics (TOG) 32, 4 (2013), 53. 